\title{
PDK1 inhibitor GSK2334470 synergizes with proteasome inhibitor MG-132 in multiple myeloma cells by inhibiting full AKT activity and increasing nuclear accumulation of the PTEN protein
}

\author{
JIN ZHANG $^{1 *}$, CHUNMEI YANG ${ }^{2 *}$, FENGPING ZHOU ${ }^{1}$ and XIAOHUI CHEN ${ }^{3}$ \\ ${ }^{1}$ Department of Hematology, Sir Run Run Shaw Hospital, College of Medicine, Zhejiang University, Hangzhou, \\ Zhejiang 310016; ${ }^{2}$ Institute of Hematology, The First Affiliated Hospital, College of Medicine, Zhejiang University, \\ Hangzhou, Zhejiang 310003; ${ }^{3}$ Department of Hematology, The Affiliated Hospital of \\ Hangzhou Normal University, Hangzhou, Zhejiang 310015, P.R. China
}

Received October 14, 2017; Accepted April 3, 2018

DOI: $10.3892 /$ or.2018.6369

\begin{abstract}
Phosphoinositide-dependent kinase 1 (PDK1) is generally active in multiple myeloma (MM) and higher expression than other hematopoietic cells, which is associated with the drug resistance and the disease progression. Previous studies have demonstrated that PDK1 can be targeted therapeutically in MM. In the present study, we examined the combination effect of GSK2334470 (GSK-470), a novel and highly specific inhibitor of PDK1, with proteasome inhibitor MG-132 in MM cell lines. GSK-470 monotherapy significantly inhibited growth of MM cell lines and induced apoptosis that was associated with the activation of both the intrinsic mitochondrial pathway and the extrinsic death receptor pathway. Moreover, GSK-470 demonstrated synergistic growth inhibitory effects with MG-132. Notably, treatment with these inhibitors resulted in an almost complete inhibition of phosphorylation of mammalian target of rapamycin on Ser2448 and Ser2481 and full activation of AKT. The combination therapy also caused an upregulation of PTEN and an increased nuclear accumulation of PTEN protein. Collectively, our results provide the rationale for novel combination treatment with PDK1 inhibitor and proteasome inhibitors to improve outcomes in patients with MM.
\end{abstract}

Correspondence to: Dr Jin Zhang, Department of Hematology, Sir Run Run Shaw Hospital, College of Medicine, Zhejiang University, 3 Qingchun East Road, Hangzhou, Zhejiang 310016, P.R. China E-mail: jeanzhang@zju.edu.cn

*Contributed equally

Key words: PDK1 inhibitor GSK2334470, proteasome inhibitor MG-132, multiple myeloma cells, inhibiting full AKT, PTEN protein

\section{Introduction}

Multiple myeloma (MM) is an incurable B-cell malignancy which accounts for approximately $2 \%$ of deaths from all cancers and $20 \%$ of deaths from all hematological malignancies. Recently, some new agents such as bortezomib, lenalidomide and autologous stem cell transplantation have been shown to significantly improve the outcomes of MM patients. However, most patients will develop refractory disease and eventually suffer a fatal relapse $(1,2)$. There are multiple mechanisms of resistance to proteasome inhibitors, such as overexpression of P-gp $(3,4)$, mutations in the proteasome $\beta 5$ subunit (PSMB5) $(5,6)$, and induction of heat shock proteins (HSPs) and autophagy (7-9). Some studies have also suggested that the activation of the phosphatidylinositol-3-kinase (PI3K)/AKT signaling pathway also resulted in resistance to bortezomib $(10,11)$. In addition, cytokines, binding with its receptor, have been reported to induce cellular proliferation, survival advantage and bortezomib resistance in MM cells via activation of a PI3K/AKT kinase cascade (12-14). For example, insulin-like growth factor-1 (IGF-1) stimulates cell growth in MM by activating AKT, especially in the context of drug resistance (12). Suppression of the IGF-1 receptor (IGF-1R) can restore sensitivity to bortezomib in MM cell lines and primary cells of patients. Combined bortezomib with IGF-1R inhibitor OSI-906 has been reported to have a greater antimyeloma activity than either one alone in vitro and in vivo (13). Suppression of AKT activation can also enhance the sensitivity of MM cells to bortezomib (15). An open-label phase 1 study indicated that an AKT inhibitor may overcome resistance to bortezomib, as a partner of combination treatment for MM (16). Therefore, alternative therapeutic strategies are needed to overcome drug resistance (17).

Phosphoinositide-dependent kinase 1 (PDK1) is suggested to act as a downstream effector in many PI3K-mediated cellular processes, which transduces multiple signaling pathways that are involved in cell survival and proliferation (18). Although definitive evidence is still lacking on whether inhibition of 
PDK1 can suppress tumorigenesis in vivo (19) it is clear that PDK1 is an intriguing target for cancer therapy. More recently, we as well as other researchers demonstrated that PDK1 inhibitors such as BX912, AR-12 and GSK2334470 (GSK-470), a novel and highly specific inhibitor of PDK1, induced growth inhibition and the induction of apoptosis, and increased the in vitro and in vivo cytotoxic effects of antimyeloma agents including chemotherapeutic drug and proteasome inhibitor bortezomibin in MM cells $(20,21)$. However, the underlying mechanism of the synergistic effects between the PDK1 inhibitor and proteasome inhibitor is still elusive. In the present study, we used a combination of GSK-470 and proteasome inhibitor MG-132 that has been previously reported to specifically target the proteasome $\beta 5$ subunit (22) to investigate the underlying mechanism responsible for the synergetic effect of the two agents.

\section{Materials and methods}

Cell culture and reagents. The RPMI8226 cell line was purchased from the American Type Culture Collection (ATCC; Rockville, MD, USA) and the ARP-1 cell line was kindly provided by Professor Cai Zhen (Zhejiang University, Hangzhou, China). The two cell lines were cultured in RPMI-1640 (HyClone Laboratories; GE Healthcare, Chicago, IL, USA) supplemented with $10 \%$ fetal bovine serum (FBS; HyClone Laboratories; GE Healthcare) at $37^{\circ} \mathrm{C}$ in an incubator with $5 \% \mathrm{CO}_{2}$. GSK-470 and MG-132 were purchased from Selleck Chemicals (Houston, TX, USA). IGF-1 was purchased from PeproTech (Rocky Hill, NJ, USA).

Cell viability assays. The inhibitory effect of GSK-470 and/or MG-132 on the proliferation of MM cell lines was examined by modified microculture tetrazolium (MTT) assay. Cells ( $2 \times 10^{4}$ cells/well) were cultured in 96-well plates and treated with GSK-470 and/or MG-132 at the indicated concentrations for $24 \mathrm{~h}$. Following this incubation, $20 \mu \mathrm{l}$ of MTT solution $(5 \mathrm{mg} / \mathrm{ml})$ was added into each well and then the plates were incubated for an additional $4 \mathrm{~h}$ at $37^{\circ} \mathrm{C}$. Following removal of the supernatant, $200 \mu \mathrm{l}$ dimethyl sulfoxide (DMSO) was added to each well, and the absorbance at a wavelength of $570 \mathrm{~nm}$ was assessed using an enzyme-linked immunosorbent assay plate reader.

Flow cytometric analysis. After treatment with GSK-470 and/or MG-132 at the indicated concentrations for $24 \mathrm{~h}, \mathrm{MM}$ cells $\left(2 \times 10^{5}\right.$ cells) were washed twice in cold phosphate-buffered saline (PBS), then the cell pellets were resuspended in $500 \mu \mathrm{l}$ of binding buffer and stained with $5 \mu \mathrm{l}$ of Annexin V-FITC and $10 \mu \mathrm{l}$ of propidium iodide (PI; Biouniquer, Suzhou, China) for $15 \mathrm{~min}$ in the dark. Cells were analyzed by FACScan flow cytometer (Becton-Dickinson, San Diego, CA, USA). Expression of green fluorescent protein (GFP) was also assessed by the FACScan flow cytometer.

Western blot analysis. For analyses of protein expression, cells treated with GSK-470 and/or MG-132 were collected by centrifuging and washed with cold PBS. Then, the cells were lysed in lysis buffer. The cytoplasm and nuclear proteins were extracted using NE-PER Nuclear and Cytoplasmic
Extraction reagents (Thermo Fisher Scientific, Inc., Waltham, MA, USA) according to the manufacturer's instructions as previously described (23). The lysate was quantified using the bicinchoninic acid (BCA) assay, and equal amounts of protein was subjected to $6-12 \%$ SDS-PAGE and transferred onto nitrocellulose membranes. After blocking with fat-free milk for $2 \mathrm{~h}$, the membranes were incubated with primary antibodies at $4^{\circ} \mathrm{C}$ overnight, and then incubated with secondary antibodies (Cell Signaling Technology, Beverly, MA, USA) for $2 \mathrm{~h}$. Antibody binding was detected by enhanced chemiluminescence using an ECL detection kit. The primary antibodies used here were as follows and all diluted to 1:1,000 for western blot experiment: Total PTEN (cat. no. 9188), phosphor-PTEN at Ser380/Thr382/383 (cat. no. 7960), total PDK1 (cat. no. 3062), phospho-PDK1 at Ser241 (cat. no. 3061), total mTOR (cat. no. 2972), phosphor-mTOR at Ser2448 (cat.no. 5536), phosphor-mTOR at Ser 2481 (cat.no. 2974), total AKT (cat. no. 9272), phosphor-AKT at Ser473 (cat. no. 4060), phosphor-AKT at Thr308 (cat. no. 13038), PI3Kp110 $\alpha$ (cat. no. 4255), poly(adenosine diphosphate-ribose) polymerase (PARP) (cat. no. 9542) and caspase-8 (cat. no. 9746), caspase-9 (cat. no. 9505) and caspase-3 (cat. no. 9662), were purchased from Cell Signaling Technology with the exception of Lamin B1 (cat. no. 12987-1-AP; Proteintech Group Inc., Chicago, IL, USA) and $\beta$-actin (cat. no. sc-69879; Santa Cruz Biotechnolgy, Santa Cruz, CA, USA).

Cellular localization studies. Cells were treated with $2 \mu \mathrm{M}$ GSK-470 and/or 200 nM MG-132 for $24 \mathrm{~h}$, washed and fixed on the $0.1 \%$ poly-L-lysine (Wuhan Boshide Bio Inc, Wuhan, China)-treated slides with $4 \%$ paraformaldehyde for $30 \mathrm{~min}$, permeabilized with $0.1 \%$ Triton X-100 and then blocked with goat serum for $30 \mathrm{~min}$. Subsequently, the slides were incubated overnight with antibody PTEN (cat. no. ab32199; Abcam, Cambridge, UK) diluted 1:100 at $4^{\circ} \mathrm{C}$, rinsed three times with PBS, and then incubated with secondary antibody: Alexa Fluor 488 (cat. no. A10468; Invitrogen; Thermo Fisher Scientific, Inc.) diluted 1:500 for $1 \mathrm{~h}$ at $37^{\circ} \mathrm{C}$. Next, the slides were repeatedly rinsed three times with PBS, and then incubated with $100 \mathrm{nM}$ rhodamine phalloidin (Cytoskeleton, Denver, CO, USA) for $30 \mathrm{~min}$. Before incubation with $0.5 \mathrm{mg} / \mathrm{ml}$ DAPI (Invitrogen; Thermo Fisher Scientific, Inc.) for $5 \mathrm{~min}$, the slides were again rinsed three times with PBS. The slides were then observed under an Olympus confocal microscope (Olympus FV1000; Olympus Corp., Tokyo, Japan).

Statistical analysis. Experimental results are presented as the mean \pm standard deviation (SD). Statistical analysis was performed by one-way analysis of variance. $\mathrm{P}<0.05$ was considered to indicate a statistically significant difference. Synergisms in the combination treatments were analyzed using CalcuSyn software (Biosoft, Cambridge, UK).

\section{Results}

GSK-470 inhibits cellular proliferation and induces apoptosis in MM cell lines. We first assessed the cytotoxic effect of GSK-470 on the human MM cell lines. As shown in Fig. 1A, both RPMI8226 and ARP-1 cell lines exhibited a sensitivity to GSK-470 in a dose-dependent manner as determined by an 


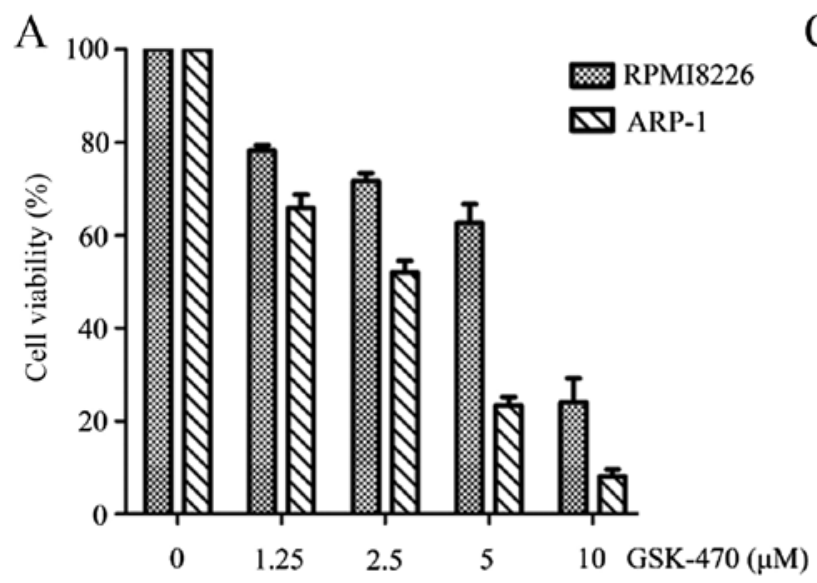

$\mathrm{C}$
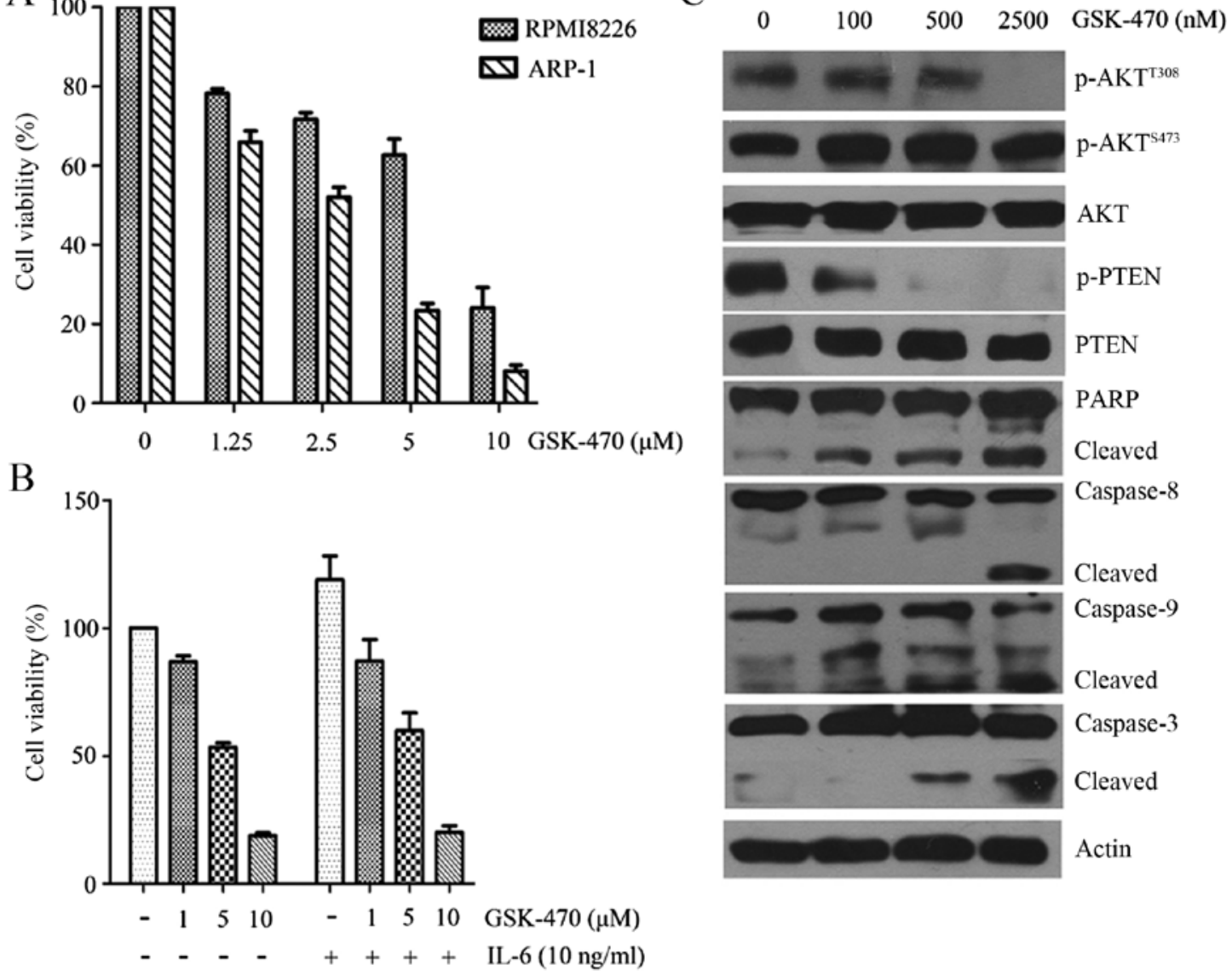

Figure 1. GSK-470 induces myeloma cell death. (A) The indicated human myeloma cell lines were cultured with increasing concentrations of GSK-470 for $48 \mathrm{~h}$ and cell viability was assessed by an MTT assay. (B) RPMI8226 cells were cultured in serum-free medium for $2 \mathrm{~h}$, followed by incubation with or without $10 \mathrm{ng} / \mathrm{ml} \mathrm{IL}-6$ for $2 \mathrm{~h}$. Then, the cells were treated with the indicated concentrations of GSK-470 for $24 \mathrm{~h}$. Cell growth was assessed using an MTT assay. Data are presented as the mean \pm SD of three independent experiments. (C) ARP-1 cells were treated with GSK-470 (100-2,500 nM) for $24 \mathrm{~h}$. Whole-cell lysates were extracted for the detection of the activation of caspase- 8 , caspase- 9 , caspase- 3 and PARP by western blot analysis. The phosphorylation and protein expression of PTEN and AKT were also examined. Actin was used as an internal standard.

MTT assay. The $50 \%$ inhibition $\left(\mathrm{IC}_{50}\right)$ values of GSK-470 at $48 \mathrm{~h}$ were $5.04 \mu \mathrm{M}$ in the RPMI8226 cells and $2.21 \mu \mathrm{M}$ in the ARP-1 cells, respectively. Since IL- 6 acts as an important mediator of cell survival, migration and drug resistance in MM (11), we next evaluated the effect of GSK-470 on RPMI8226 in the presence of exogenous IL-6. RPMI8226 cells were incubated in FBS-free RPMI-1640 medium containing 1-10 $\mu \mathrm{M}$ GSK-470 and $100 \mathrm{ng} / \mathrm{ml}$ IL-6. Stimulation by IL-6 increased cell growth of myeloma cells, but GSK-470 was able to suppress this stimulation (Fig. 1B). Following treatment of ARP-1 cells with GSK-470 at various concentrations $(100-2,500 \mathrm{nM})$ for $24 \mathrm{~h}$, we revealed that GSK-470 triggered a time-dependent cleavage of caspase- $8,-9$ and -3 , followed by PARP cleavage (Fig. 1C). These data indicated that GSK-470 induced cytotoxicity and apoptosis in myeloma cells.

GSK-470 significantly inhibited the phosphorylation of AKT at Thr308 (Fig. 1C). Since inhibition of PTEN phosphorylation is important for PTEN protein stability to proteasome-mediated degradation (24), we next examined the effect of GSK-470 on the phosphorylation of PTEN in ARP-1 cells. GSK-470 induced a notable inhibition of phosphor-PTEN at Ser380 and Thr382/383 (Fig. 1C), which may accelerate the proteasome-mediated degradation of PTEN (24).
MG-132 sensitizes MM cells to GSK-470-mediated cell death. Since a previous study reported that the combined use of PDK1 inhibitor BX-912 and bortezomib exerts synergistic effect in MM cells (21), we examined whether the sensitivity of RPMI8226 cells to GSK-470 was enhanced by MG-132. For this purpose, we treated RPMI8226 cells with a series of concentrations of GSK-470 and MG-132 either alone or in combination for $24 \mathrm{~h}$ and assessed cell viability using an MTT assay. As revealed in Fig. 2A, co-treatment with GSK-470 and MG-132 led to significantly lower cell viability than either GSK-470 or MG-132 treatment alone. The combination index values were $<1$, which indicated a synergistic effect between GSK-470 and MG-132 (Fig. 2B). Flow cytometric analysis revealed an enhanced apoptosis of cells following exposure to treatment with GSK-470 plus MG-132 (Fig. 2C). We also evaluated the cytotoxicity triggered by GSK-470 and MG-132 against primary myeloma cells. The myeloma cells from a patient newly diagnosed with $\mathrm{MM}$ were treated with GSK-470 $(5 \mu \mathrm{M})$ and/or MG-132 (400 nM) for the detection of cell viability and apoptosis. Combination of GSK-470 with MG-132 resulted in an enhanced cytotoxicity against primary myeloma cells and produced more apoptotic cells (Fig. 3). 


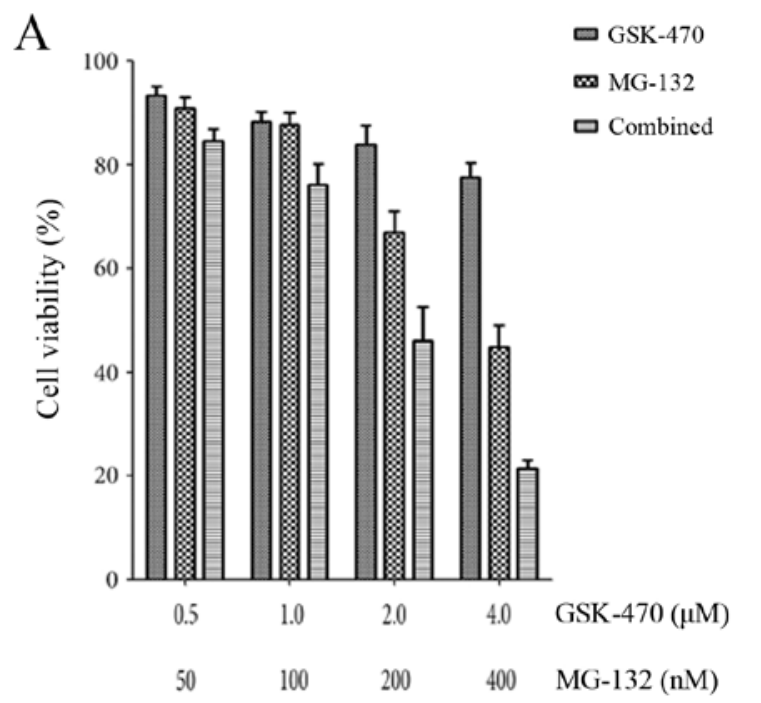

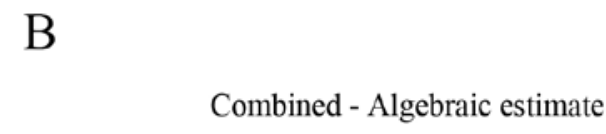

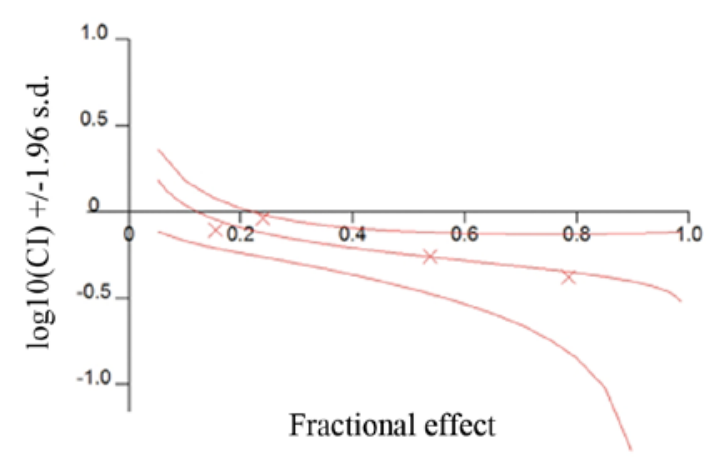

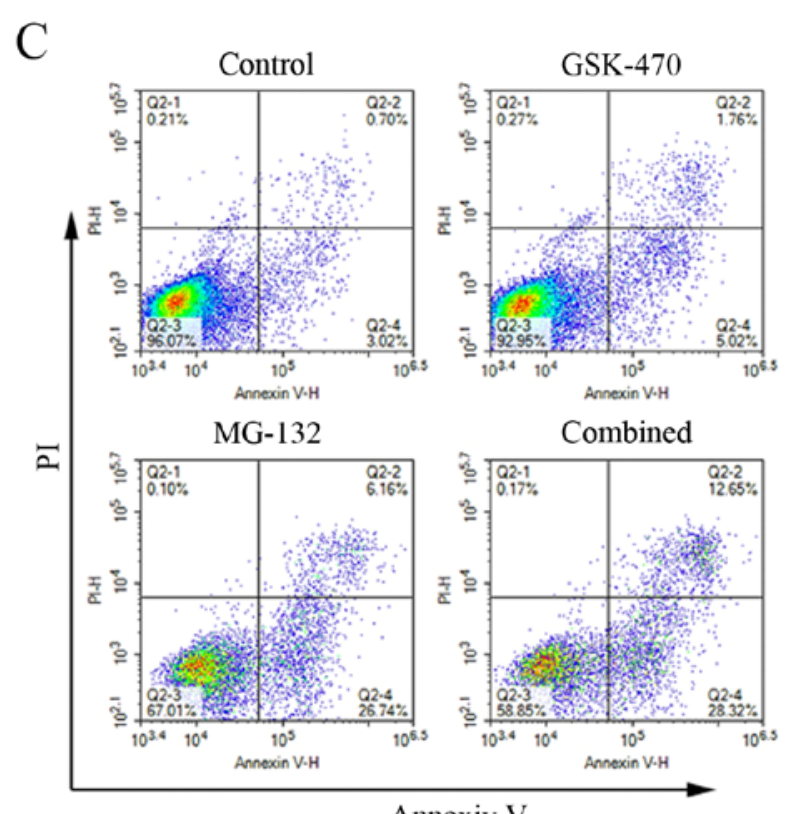

Annexiv V

Figure 2. Synergistic cytotoxicity to MM cells of GSK-470 in combination with MG-132. (A) RPMI8226 cells were treated with increasing concentrations of GSK-470 and/or MG-132 for $24 \mathrm{~h}$, and cell viability was determined by MTT metabolization. Data are presented as the means \pm SD of 3 independent experiments. (B) The combination index values were calculated as described in Materials and methods. (C) RPMI8226 cells were treated with GSK-470 ( $2 \mu \mathrm{M})$, MG-132 (200 nM) or GSK-470 plus MG-132 for $24 \mathrm{~h}$, and then apoptotic cells were determined by flow cytometry after dual-staining of cells with Annexin V and propidium iodide $(\mathrm{PI})$.

GSK-470 combined with MG-132 results in almost complete inhibition of the activity of AKT as well as $m$ TORC1/mTORC2. In order to characterize the cytotoxicity of GSK-470 combined with MG-132 against MM cells, we examined the alteration of the apoptotic pathway induced by the co-treatment. Western blot analysis indicated that RPMI8226 cells treated with GSK-470 or MG-132 alone demonstrated a weak activation of both the extrinsic and intrinsic apoptosis pathway, indicated by cleavage of caspase-8, caspase-9, caspase-3 and PARP. In contrast, an enhanced activation of cleaved caspase molecules was observed in the myeloma cells treated with the combination therapy (Fig. 4A).

The PI3K/AKT/mTOR signaling pathway is frequently hyperactivated in MM cells, which is associated with resistance to antimyeloma agents $(20,25)$. Our data indicated that
GSK-470 inhibited PI3K p110 $\alpha$ protein expression. However, co-treatment with GSK-470 and MG-132 led to a markedly enhanced inhibition of PI3K p110 $\alpha$ (Fig. 4B). Western blot analysis also indicated that GSK-470 decreased phosphorylation of AKT at Thr308 and phosphorylation of mTOR on Ser2448, a downstream molecule of AKT, consistent with the notion that Thr308 residues of AKT are the main effectors of PDK1 in cancer cells (20). Additionally, MG-132 exhibited inhibitory effects on the phosphorylation of AKT at Ser473 and phosphorylation of mTOR at Ser2481, a marker for mTORC2 activity. Notably, the combination therapy led to almost complete elimination of phosphorylated AKT (Ser473/Thr308) and activity of mTORC1 as well as mTORC2. Furthermore, significantly upregulated expression of PTEN was observed in the MG-132-treated cells compared to the untreated cells, which was consistent with a recent observation which revealed 

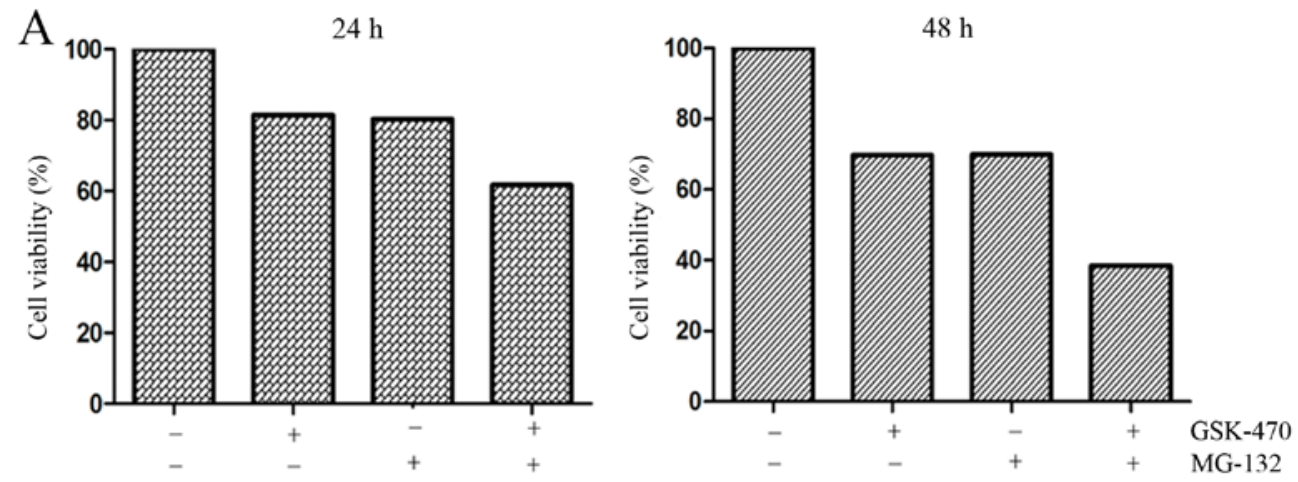

$\mathrm{B}$

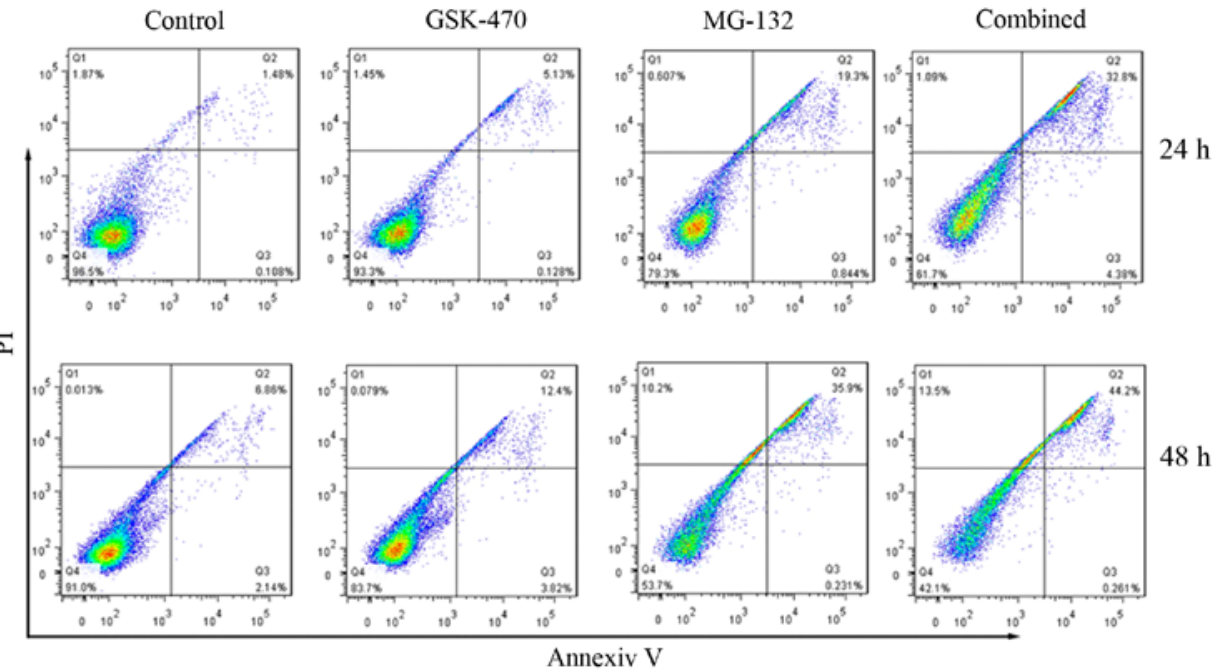

Figure 3. The antitumor effect of GSK-470 alone or combined with MG132 on primary myeloma cells. (A) Primary cells were obtained from a 65-year-old patient with newly diagnosed MM who presented with $81 \%$ of abnormal plasma cells in bone marrow. The primary myeloma cells were treated with GSK-470 $(5 \mu \mathrm{M})$, MG132 (400 nM) or GSK-470 combined with MG-132 for 24 and $48 \mathrm{~h}$, respectively. Cell viability was determined by an MTT assay. Data are presented as the means \pm SD of three independent experiments. (B) The primary myeloma cells were cultured in similar conditions for 24 and $48 \mathrm{~h}$, respectively. Induction of apoptosis was examined by Annexin V and PI double-staining.
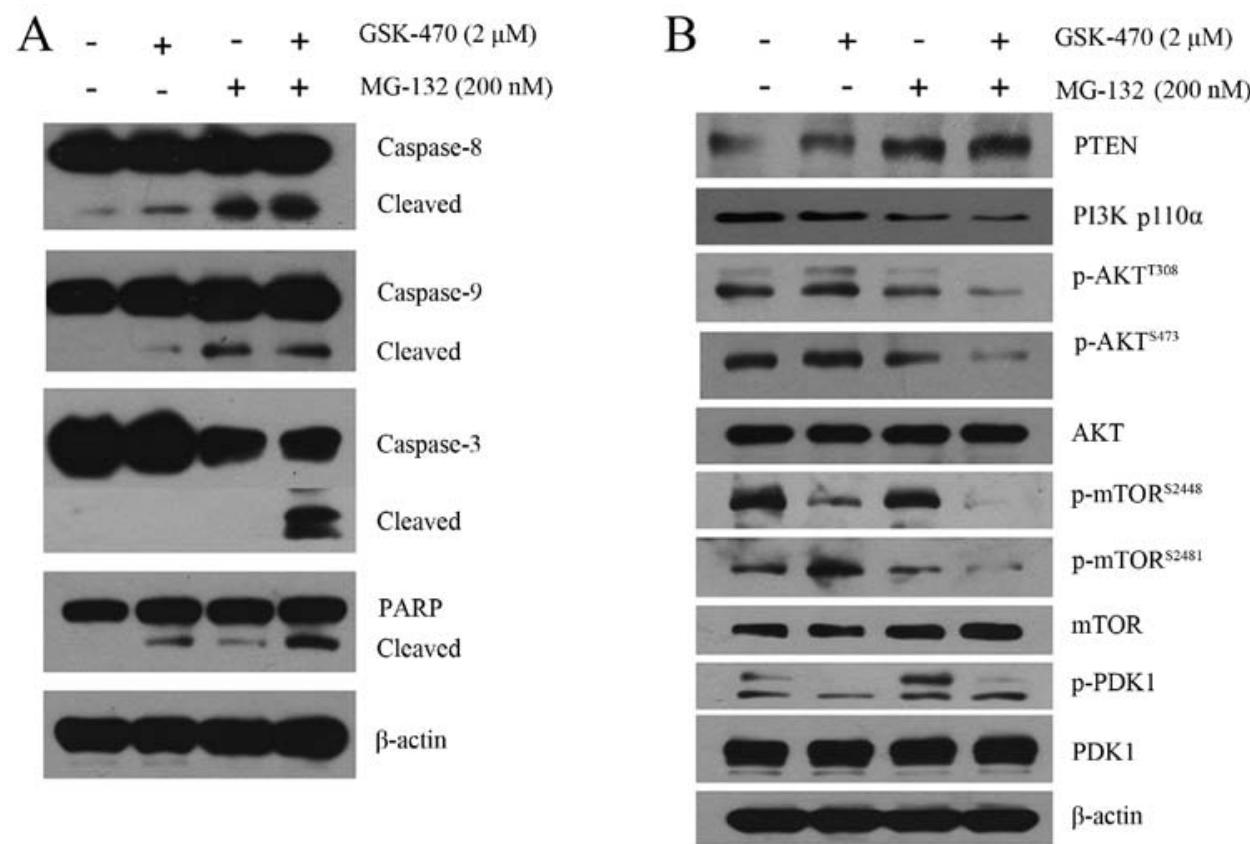

Figure 4. Apoptosis induced by the combination of GSK-470 and MG-132 is associated with almost complete inhibition of phosphorylation of AKT and mTOR. (A) RPMI8226 cells were treated with $2 \mu$ M GSK- 470 and/or $200 \mathrm{nM}$ MG-132 for $24 \mathrm{~h}$. Cleavage of PARP, caspase-3, caspase- 8 and caspase-9 were analyzed by western blot analysis. (B) RPMI8226 cells were treated with $2 \mu \mathrm{M}$ GSK-470 and/or $200 \mathrm{nM}$ MG-132 for $24 \mathrm{~h}$. Whole-cell lysates were subjected to western blotting to determine the level of phosphorylation and protein expression of PDK1, AKT and mTOR. The expression of PTEN and PI3K p110 $\alpha$ was also evaluated. $\beta$-actin was used as a protein loading control. 
A Control GSK-470 MG-132 Combined

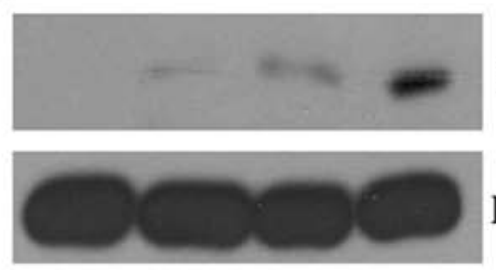

1.0

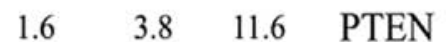

Nucleus
Control GSK-470 MG-132 Combined

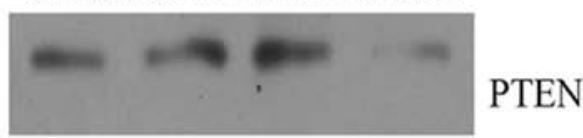

Actin
1.0
$\begin{array}{ll}1.1 & 1.7\end{array}$
0.4 PTEN
Cytoplasm

B
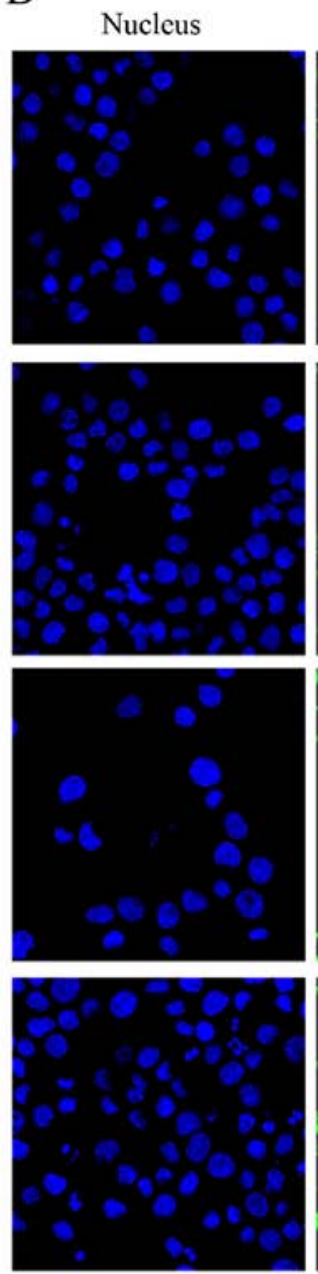

PTEN
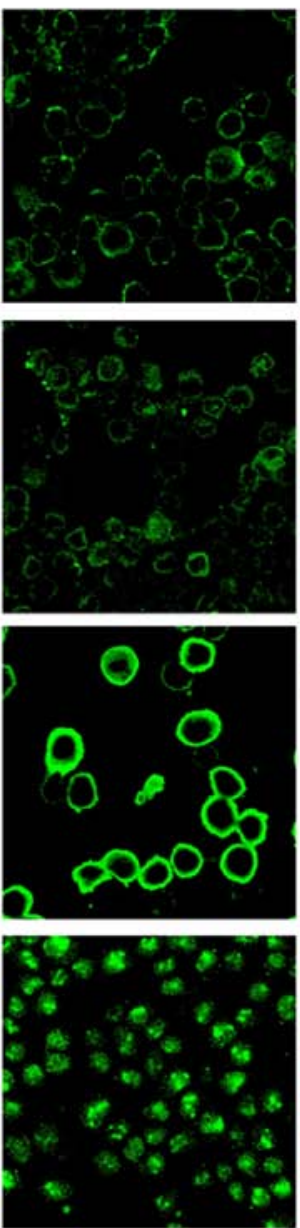

Actin
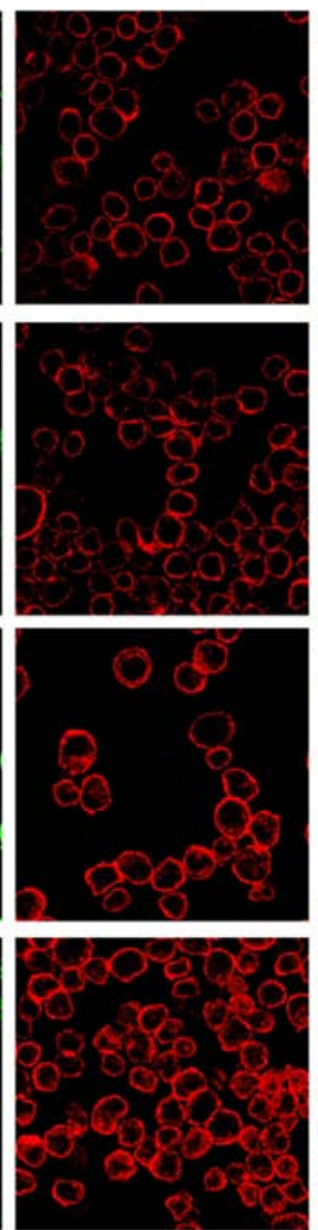

Merged
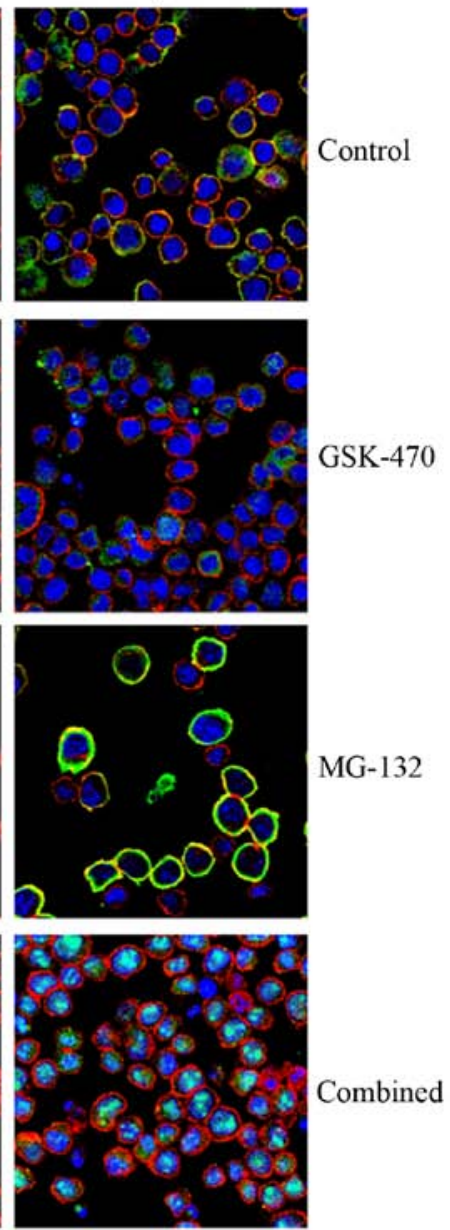

Figure 5. Combination of GSK-470 with MG-132 results in an increased nuclear accumulation of PTEN protein. (A) After treatment with GSK-470 (2 $\mu$ M) and/or MG-132 $(200 \mathrm{nM})$ for $24 \mathrm{~h}$, nuclear and cytoplasmic extracts of RPMI8226 cells were prepared to examine the level of PTEN by western blotting. (B) In addition, color confocal microscopy analysis of PTEN protein (green) and $\beta$-actin (red) was performed. Cell nuclei were stained in blue (DAPI). A representative area is shown.

that bortezomib increased PTEN expression in drug-resistant breast cancer cells (26).

Increased PTEN accumulation in the nucleus may play a crucial role in the synergistic effect between GSK-470 and $M G-132$. Recent studies demonstrated that in the nucleus PTEN exerts an important tumor-suppressive function (27). In the present study, PTEN expression was slightly elevated in nucleus of cells treated with GSK-470 compared to the untreated control. Whereas, a higher level of PTEN was found in the nuclear protein extracts of RPMI8226 cells treated with MG-132, but not in the cytoplasmic extracts. Furthermore, combination of GSK-470 and MG-132 resulted in a significantly increased level of PTEN protein in the nucleus but decreased PTEN protein expression in the cytoplasm (Fig. 5A). To confirm the subcellular localization of PTEN in MM cells, confocal microscopy studies were performed (Fig. 5B). Intense staining of PTEN was observed at the nuclear periphery and in the cytoplasm of MG-132-treated cells. Notably, PTEN was found mainly in the nucleus when cells were treated with the 
combination therapy. Collectively, these findings demonstrated that combination of PDK1 inhibitor GSK-470 and proteasome inhibitor MG-132 resulted in the nuclear accumulation of PTEN.

\section{Discussion}

Targeting PDK1 may represent a promising therapeutic approach for MM since PDK1 is implicated in signaling pathways frequently activated in cancer, such as PI3K/AKT, Ras/MAPK and Myc (28). It has been reported that PDK1 is active in myeloma cells in a majority of MM patients, which is associated with disease progression and resistance to treatment (21). We as well as other researchers have previously revealed that the PDK1 inhibitor is very effective at killing myeloma cells $(20,21)$. In the present study, we set out to characterize the potential antimyeloma activity of combined GSK-470 with MG-132, as well as to investigate the underlying mechanism by which GSK470 and MG-132 synergize to kill myeloma cells. Our findings demonstrated a synergism in vitro, demonstrating the effectiveness of targeting both PDK1 and a proteasome in this model. The combination therapy resulted in an enhanced apoptosis and activation of both caspase- 8 and -9 followed by downstream activation of caspase- 3 and PARP, suggesting that the two main pathways of procaspase activation (the intrinsic mitochondrial pathway and the extrinsic death receptor pathway) are involved.

AKT kinase is a well-studied viability-promoting effector molecule, which is activated in MM cell lines (29) and in the tumors of patients (30). AKT signaling mediates MM cell resistance to chemotherapeutic agents such as dexamethasone, melphalan, vincristine, and bortezomib (31). IGF-1 is a critical growth factor in MM and confers drug resistance also by activating AKT (14). Our data demonstrated that exogenous IGF-1 did not reverse the growth inhibition induced by GSK-470 and that GSK-470 inhibited phosphor-PDK1, consequently suppressing the phosphorylation of AKT at Thr308, but failed to suppress phosphor-AKT on Ser473. These results are consistent with the finding that PDK1 directly phosphorylates Thr308 residues of AKT (32), but requires mTOR complex 2 (mTORC2)-induced AKT phosphorylation on Ser473 for full activation of AKT (33). Notably, we demonstrated that combination of GSK-1 and MG-132 resulted in potent suppression of not only phosphor-AKT at Thr308 and Ser473 but also phosphor-mTOR at Ser2448 and Ser2481. Collectively, these results revealed that the combination of GSK-470 with MG-132 may be particularly useful to drug resistance.

Loss of function of the PTEN gene as well as increased expression of both PI3K and AKT are frequent events in cancers. In addition to mutation or deletion in PTEN itself, epigenetic silencing by gene promoter methylation and alterations of miRNA such as miR-221/222, miR-19a and miR-22 are also involved in cancer (34-37). In MM, the aberrant expression of PTEN may be associated with disease progression (38). It is well known that normal phosphorylation of PTEN in its C-terminal non-catalytic regulatory domain is important for PTEN protein stability to proteasome-mediated degradation $(24,39)$. Our data indicated that GSK-470 significantly inhibited the phosphorylation of PTEN at Ser380/Thr382/383, which could lead to rapid degradation of PTEN. However, we revealed that the upregulation of PTEN was observed in RPMI8226 cells treated with MG-132, a novel proteasome inhibitor, suggesting that MG-132 treatment decreased protein degradation of PTEN. This result is in accordance with the observation that MG-132 inhibited the degradation of both PTEN wild-type and mutant (24), and is consistent with a previous study which revealed that bortezomib increased PTEN expression and enhanced trastuzumab-induced growth inhibition in trastuzumab-resistant breast cancer cells (26). Notably, our findings indicated that co-treatment of GSK-470 and MG-132 significantly induced an increased nuclear localization of the PTEN protein in MM cells. Based on previous studies demonstrating that nuclear PTEN plays a significant role in the maintenance of genomic stability through the modulation of DNA repair, chromosomal segregation, and cell cycle arrest (40) and that PTEN nuclear localization is regulated by oxidative stress and mediates p53-dependent tumor suppression (41), our results revealed that increased nuclear localization of the PTEN protein elicited by the combination therapy may contribute to the synergistic effect on MM cell death.

In summary, the data presented in this study demonstrated the synergy between GSK-470 and MG-132 in MM. Mechanistically, treatment with these inhibitors significantly suppressed full activity of AKT and mTORC1/mTORC2 activity. In particular this combination therapy upregulated PTEN and resulted in an increased nuclear accumulation of the PTEN protein. Collectively, our data may provide the framework for utilizing the PDK1 inhibitor in combination with a proteasome inhibitor to enhance anti-MM activities.

\section{Acknowledgements}

The authors thank all the investigators, including laboratory technicians in this study.

\section{Funding}

The present study was supported by the National Natural Science Foundation of China (grant nos. 81370645 and 81670178), The National Key Research and Development Program of China (no. 2016YFC090150X), the Funds of the Science of Zhejiang Province (grant no. Y15H080001) and the Special Scientific Construction Research Funds of the National Chinese Medicine Clinical Research Center, SATCM (grant no. JDZX2015113).

\section{Availability of data and materials}

The datasets used and/or analyzed during the current study are available from the corresponding author on reasonable request.

\section{Authors' contributions}

CMY performed the experiments, JZ and CMY made substantial contributions to conception and design of data, analysis and interpretation of data. FPZ and XHC have been involved in drafting the manuscript and revising it critically 
for important intellectual content. All authors read and approved the manuscript and agree to be accountable for all aspects of the research in ensuring that the accuracy or integrity of any part of the work are appropriately investigated and resolved.

\section{Ethics approval and consent to participate}

This study did not involve human participants, identifiable human data or human tissue which needed a statement on ethics approval and consent.

\section{Consent for publication}

This study did not involve research with patients and thus informed consent for publication was not required.

\section{Competing interests}

The authors declare that they have no conflict of competing interests.

\section{References}

1. Naymagon L and Abdul-Hay M: Novel agents in the treatment of multiple myeloma: A review about the future. J Hematol Oncol 9: $52,2016$.

2. Wang Y, Sanchez L, Siegel DS and Wang ML: Elotuzumab for the treatment of multiple myeloma. J Hematol Oncol 9: 55, 2016

3. Gutman D, Morales AA and Boise LH: Acquisition of a multidrug-resistant phenotype with a proteasome inhibitor in multiple myeloma. Leukemia 23: 2181-2183, 2009.

4. Ao L, Wu Y, Kim D, Jang ER, Kim K, Lee DM, Kim KB and Lee W: Development of peptide-based reversing agents for P-glycoprotein-mediated resistance to carfilzomib. Mol Pharm 9: 2197-2205, 2012.

5. Fuchs D, Berges C, Opelz G, Daniel V and Naujokat C: Increased expression and altered subunit composition of proteasomes induced by continuous proteasome inhibition establish apoptosis resistance and hyperproliferation of Burkitt lymphoma cells. J Cell Biochem 103: 270-283, 2008.

6. Rückrich T, Kraus M, Gogel J, Beck A, Ovaa H, Verdoes M, Overkleeft HS, Kalbacher H and Driessen C: Characterization of the ubiquitin-proteasome system in bortezomib-adapted cells. Leukemia 23: 1098-1105, 2009.

7. Bustany S, Cahu J, Descamps G, Pellat-Deceunynck C and Sola B: Heat shock factor 1 is a potent therapeutic target for enhancing the efficacy of treatments formultiple myeloma with adverse prognosis. J Hematol Oncol 8:40, 2015.

8. Richardson PG, Schlossman RL, Alsina M, Weber DM, Coutre SE, Gasparetto C, Mukhopadhyay S, Ondovik MS, Khan M, Paley CS, et al: PANORAMA 2: Panobinostat in combination with bortezomib and dexamethasone in patients with relapsed and bortezomib-refractory myeloma. Blood 122: 2331-2337, 2013.

9. Riz I, Hawley TS and Hawley RG: KLF4-SQSTM1/p62-associated prosurvival autophagy contributes to carfilzomib resistance in multiple myeloma models. Oncotarget 6: 14814-14831, 2015.

10. Yu W, Chen Y, Xiang R, Xu W, Wang Y, Tong J, Zhang N, $\mathrm{Wu}$ Y and Yan H: Novel phosphatidylinositol 3-kinase inhibitor BKM120 enhances the sensitivity of multiple myeloma to bortezomib and overcomes resistance. Leuk Lymphoma 58: 428-437, 2017.

11. Hideshima T, Mitsiades C, Tonon G, Richardson PG and Anderson KC: Understanding multiple myeloma pathogenesis in the bone marrow to identify new therapeutic targets. Nat Rev Cancer 7: 585-598, 2007.

12. Bieghs L, Johnsen HE, Maes K, Menu E, Van Valckenborgh E, Overgaard MT, Nyegaard M, Conover CA, Vanderkerken K and De Bruyne E: The insulin-like growth factor system in multiple myeloma: Diagnostic and therapeutic potential. Oncotarget 7: 48732-48752, 2016.
13. Kuhn DJ, Berkova Z, Jones RJ, Woessner R, Bjorklund CC, Ma W, Davis RE, Lin P, Wang H, Madden TL, et al: Targeting the insulin-like growth factor-1 receptor to overcome bortezomib resistance in preclinical models of multiple myeloma. Blood 120: 3260-3270, 2012.

14. Jernberg-Wiklund $\mathrm{H}$ and Nilsson $\mathrm{K}$ : Control of apoptosis in human multiple myeloma by insulin-like growth factor I (IGF-I). Adv Cancer Res 97: 139-165, 2007.

15. Hideshima T, Catley L, Yasui H, Ishitsuka K, Raje N, Mitsiades C, Podar K, Munshi NC, Chauhan D, Richardson PG and Anderson KC: Perifosine, an oral bioactive novel alkylphospholipid, inhibits Akt and induces in vitro and in vivo cytotoxicity in human multiple myeloma cells. Blood 107: 4053-4062, 2006.

16. Spencer A, Yoon SS, Harrison SJ, Morris SR, Smith DA, Brigandi RA, Gauvin J, Kumar R, Opalinska JB and Chen C: The novel AKT inhibitor afuresertib shows favorable safety, pharmacokinetics, and clinical activity in multiple myeloma. Blood 124: 2190-2195, 2014.

17. Liu L, Zhao N, Xu W, Sheng Z and Wang L: Pooled analysis of the reports of carfilzomib, panobinostat, and elotuzumab combinations inpatients with refractory/relapsed multiple myeloma. J Hematol Oncol 9: 54, 2016.

18. Hu T, Li C, Wang L, Zhang Y, Peng L, Cheng H, Chu Y, Wang W, Ema H, Gao Y, et al: PDK1 plays a vital role on hematopoietic stem cell function. Sci Rep 7: 4943, 2017.

19. Du J, Yang M, Chen S, Li D, Chang Z and Dong Z: PDK1 promotes tumor growth and metastasis in a spontaneous breast cancer model. Oncogene 35: 3314-3323, 2016.

20. Yang C, Huang X, Liu H, Xiao F, Wei J, You L and Qian W: PDK1 inhibitor GSK2334470 exerts antitumor activity in multiple myeloma and forms a novel multitargeted combination with dual mTORC1/C2 inhibitor PP242. Oncotarget 8: 39185-39197, 2017.

21. Chinen Y, Kuroda J, Shimura Y, Nagoshi H, Kiyota M, Yamamoto-Sugitani M, Mizutani S, Sakamoto N, Ri M, Kawata E, et al: Phosphoinositide protein kinase PDPK1 is a crucial cell signaling mediator in multiple myeloma. Cancer Res 74: 7418-7429, 2014.

22. Crawford LJ, Walker B, Ovaa H, Chauhan D, Anderson KC, Morris TC and Irvine AE: Comparative selectivity and specificity of the proteasome inhibitors BzLLLCOCHO, PS-341, and MG-132. Cancer Res 66: 6379-6386, 2006.

23. He W, Ye X, Huang X, Lel W, You L, Wang L, Chen X and Qian W: Hsp90 inhibitor, BIIB021, induces apoptosis and autophagy by regulating mTOR-Ulk1 pathway in imatinib-sensitive and -resistant chronic myeloid leukemia cells. Int J Oncol 48: 1710-1720, 2016.

24. Torres J and Pulido R: The tumor suppressor PTEN is phosphorylated by the protein kinase CK2 at its $\mathrm{C}$ terminus. Implications for PTEN stability to proteasome-mediated degradation. J Biol Chem 276: 993-998, 2001.

25. Malek E and Driscoll JJ: High throughput chemical library screening identifies a novel $\mathrm{p} 110-\delta$ inhibitor that potentiates the anti-myeloma effect of bortezomib. Oncotarget 7: 38523-38538, 2016.

26. Fujita T, Doihara H, Washio K, Kawasaki K, Takabatake D, Takahashi H, Tsukuda K, Ogasawara Y and Shimizu N: Proteasome inhibitor bortezomib increases PTEN expression and enhances trastuzumab-induced growth inhibition in trastuzumab-resistant cells. Anticancer Drugs 17: 455-462, 2006.

27. Bononi A and Pinton P: Study of PTEN subcellular localization. Methods 77-78: 92-103, 2015.

28. Gagliardi PA, Puliafito A and Primo L: PDK1: At the crossroad of cancer signaling pathways. Semin Cancer Biol 48: 27-35, 2018.

29. Tu Y, Gardner A and Lichtenstein A: The phosphatidylinositol 3-kinase/AKT kinase pathway in multiple myeloma plasma cells: Roles in cytokine-dependent survival and proliferative responses. Cancer Res 60: 6763-6770, 2000.

30. Hsu J, Shi Y, Krajewski S, Renner S, Fisher M, Reed JC, Franke TF and Lichtenstein A: The AKT kinase is activated in multiple myeloma tumor cells. Blood 98: 2853-2855, 2001.

31. Keane NA, Glavey SV, Krawczyk J and O'Dwyer M: AKT as a therapeutic target in multiple myeloma. Expert Opin Ther Targets 18: 897-915, 2014.

32. Sarbassov DD, Guertin DA, Ali SM and Sabatini DM: Phosphorylation and regulation of Akt/PKB by the rictor-mTOR complex. Science 307: 1098-1101, 2005. 
33. Williams MR, Arthur JS, Balendran A, van der Kaay J, Poli V, Cohen P and Alessi DR: The role of 3-phosphoinositide-dependent protein kinase 1 in activating AGC kinases defined in embryonic stem cells. Curr Biol 10: 439-448, 2000.

34. Piras G, Monne M, Palmas AD, Calvisi A, Asproni R, Vacca F, Pilo L, Gabbas A and Latte G: Methylation analysis of the phosphates and tensin homologue on chromosome 10 gene (PTEN) in multiple myeloma. Clin Epigenetics 6: 16, 2014.

35. Naidu S, Magee P and Garofalo M: MiRNA-based therapeutic intervention of cancer. J Hematol Oncol 8: 68, 2015.

36. Di Martino MT, Gullà A, Cantafio ME, Lionetti M, Leone E Amodio N, Guzzi PH, Foresta U, Conforti F, Cannataro M, et al: In vitro and in vivo anti-tumor activity of miR-221/222 inhibitors in multiple myeloma. Oncotarget 4: 242-255, 2013.

37. Zhang X, Chen Y, Zhao P, Zang L, Zhang Z and Wang X: MicroRNA-19a functions as an oncogene by regulating PTEN/AKT/pAKT pathway in myeloma. Leuk Lymphoma 58: 932-940, 2017.
38. Wang SY, Hao HL, Deng K, Li Y, Cheng ZY, Lv C, Liu ZM, Yang $\mathrm{J}$ and Pan L: Expression levels of phosphatase and tensin homolog deleted on chromosome 10 (PTEN) and focal adhesion kinase in patients with multiple myeloma and their relationship to clinical stage and extramedullary infiltration. Leuk Lymphoma 53: 1162-1168, 2012.

39. Fragoso R and Barata JT: Kinases, tails and more: Regulation of PTEN function by phosphorylation. Methods 77-78: 75-81, 2015.

40. Ho J, Bassi C and Stambolic V: Characterization of nuclear PTEN and its post translational modifications. Methods 77-78: 104-111, 2015.

41. Chang CJ, Mulholland DJ, Valamehr B, Mosessian S, Sellers WR and Wu H: PTEN nuclear localization is regulated by oxidative stress and mediates p53-dependent tumor suppression. Mol Cell Biol 28: 3281-3289, 2008. 